\title{
COVID-19 en pacientes con patologías hematológicas
}

\author{
Gilberto Barranco-Lampón*, Yubelka Baltodano-Martínez, Carlos Martínez-Murillo, \\ Humberto Castellanos-Sinco, Christian Ramos-Peñafiel, Francisco Zazueta-Pozos, \\ Manuel de la Mora-Estrada, Katherine Rodríguez-Fuentes, Efreen Montaño-Figueroa, \\ Karen Pellón-Téllez, Juan Muñoz-Vega, Mario Tapia-Bravo y Claudia Tellería-Arispe \\ Departamento de Hematología, Unidad 111D, Hospital General de México Eduardo Liceaga, Ciudad de México, México
}

\section{Resumen}

Los informes iniciales sugirieron que los pacientes con antecedentes o malignidad activa podrían tener un mayor riesgo de contraer el coronavirus 2 del síndrome respiratorio agudo grave (SARS-CoV-2) y desarrollar complicaciones relacionadas con la enfermedad por coronavirus 2019 (COVID-19). Pacientes con patologías hematológicas benignas y malignas pueden estar inmunocomprometidos por los efectos de la terapia antineoplásica, medicamentos de apoyo como los esteroides y las propiedades inmunosupresoras del cáncer en sí. También podrían tener una respuesta inmunitaria aumentada a la infección secundaria a fármacos inmunomoduladores. Se espera que la COVID-19, causada por el SARS-CoV-2, sea una infección devastadora en muchos pacientes con enfermedades hematológicas. En México se reportaron los primeros casos confirmados el 1 de marzo de 2020; en nuestro servicio de hematología el primer caso reportado y confirmado fue en abril de 2020. Realizamos un estudio de serie de casos de 33 pacientes hospitalizados con patologías benignas y malignas que desarrollaron COVID-19. Las tasas de casos de COVID-19 en sujetos hospitalizados con patologías hematológicas fue del $15.7 \%$. La mortalidad por COVID-19 fue del 54.54\%. En pacientes con patologías hematológicas parece deberse principalmente a que los pacientes con cáncer activo sin respuesta completa que recibieron quimioterapia citotóxica u otro tratamiento contra el cáncer tienen un mayor riesgo de mortalidad por la COVID-19 en comparación con aquellos que no reciben tratamiento activo, pacientes de novo sin quimioterapia, pero en estadios avanzados de la enfermedad con comorbilidades y asociadas principalmente con coinfecciones bacterianas.

PALABRAS CLAVE: COVID 19. Hematología. Neoplasias hematológicas. Hematooncología.

\section{COVID-19 in patients with hematological diseases}

\begin{abstract}
Initial reports suggested that patients with a history or active malignancy may be at increased risk of contracting severe acute respiratory syndrome coronavirus 2 (SARS-CoV-2) and developing complications related to coronavirus disease 2019 (COVID-19). Patients with benign and malignant hematological pathologies may be immunocompromised by the effects of antineoplastic therapy, supportive medications such as steroids, and the immunosuppressive properties of the cancer itself. They may also have an increased immune response to infection secondary to immunomodulatory drugs. COVID-19, caused by SARS-CoV-2, is expected to be an infection devastating in many patients with hematologic diseases. The first confirmed cases in Mexico were on March 1, 2020; In our hematology service, the first case reported and confirmed was in April 2020. We conducted a case series study of 33 hospitalized patients with benign and malignant pathologies that developed COVID-19. The COVID-19 case rates in hospitalized subjects with hematological pathologies was $15.7 \%$. The mortality from COVID-19
\end{abstract}

Correspondencia:

*Gilberto Barranco-Lampon

E-mail: drgilbalampon@gmail.com
Fecha de recepción: 09-09-2020

Fecha de aceptación: 09-02-2021

DOI: $10.24875 / G M M . M 21000488$
Gac Med Mex. 2021;157(Supl 3):S3-S9

Disponible en PubMed

www.gacetamedicademexico.com

0016-3813/৫ 2021 Academia Nacional de Medicina de México, A.C. Publicado por Permanyer. Este es un artículo open access bajo la licencia CC BY-NC-ND (http://creativecommons.org/licenses/by-nc-nd/4.0/). 
was 54.54\%. In patients with hematological pathologies it seems to be mainly due to the fact that patients with active cancer without a complete response who received cytotoxic chemotherapy or other anti-cancer treatment cancer have a higher risk of mortality from COVID-19 compared to those who do not receive active treatment, patients de novo without chemotherapy, but in advanced stages of the disease with comorbidities and associated mainly with bacterial coinfections.

KEY WORDS: Hematology. COVID-19. Hematology. Hematological diseases.

\section{Introducción}

La enfermedad por coronavirus 2019 (COVID-19) surgió a fines de 2019 en Wuhan, China, y se ha propagado rápidamente. A medida que la infección se ha generalizado, ha aumentado la preocupación por la influencia de la COVID-19 en pacientes con cánceres hematológicos y patologías benignas hematológicas ${ }^{1-7}$.

Entre los pacientes con infección por coronavirus 2 del síndrome respiratorio agudo grave (SARS-CoV-2), la neumonía, la insuficiencia respiratoria y el síndrome de dificultad respiratoria aguda son complicaciones frecuentes. Aunque la fisiopatología subyacente a la COVID-19 grave sigue siendo poco conocida, la evidencia acumulada argumenta a favor del síndrome hiperinflamatorio, que causa la liberación de citocinas fulminantes y fatales asociadas con la gravedad de la enfermedad y el mal resultado $0^{1-3,7,8-11}$.

Se espera que la COVID-19, causado por el SARS-CoV-2, sea una infección devastadora en muchos pacientes con enfermedades hematológicas ${ }^{2-4}$.

Los pacientes que generalmente tratamos tienen un alto riesgo de infecciones oportunistas debido a la insuficiencia de la médula ósea y la supresión inmunitaria producida por los medicamentos dirigidos a la función de la médula ósea, o a ambos ${ }^{2-4,9,8,12}$.

Existen pocos datos sobre el riesgo de desarrollar COVID-19 en personas hospitalizadas con patologías hematológicos. Muchas, si no la mayoría de las personas con patologías hematológicas benignas y malignas, reciben medicamentos contra el cáncer con función supresora de la médula ósea o tienen cánceres del sistema inmunitario y tienen un riesgo sustancial de infecciones adquiridas en la comunidad y en el hospital $2-4,9,8,12,13$.

La tasa de letalidad de casos específicos de cáncer que la ASCO (American Society of Clinical Oncology) puede identificar figura en un informe de la misión conjunta Organización Mundial de la Salud (OMS)China sobre la enfermedad del coronavirus publicado el 28 de febrero de 2020. Este informe indica que en China, a partir de los datos corte (20 de febrero), la tasa de letalidad de los pacientes con cáncer como condición comórbida e infección confirmada por laboratorio fue del $7.6 \%$. Esto se compara con: un $3.8 \%$ general, sin afección comórbida un 1.4\%, con enfermedad cardiovascular un $13.2 \%$, con diabetes un $9.2 \%$, con hipertensión un $8.4 \%$ y con enfermedad respiratoria crónica un $8.0 \%$ 3,4,9,10,12,13.

$\mathrm{He}$, et al. ${ }^{2}$ informaron sobre un estudio de cohorte de 128 pacientes con cánceres hematológicos con COVID-19 en comparación con un conjunto contemporáneo de 226 proveedores de atención médica con COVID-19 como grupo de comparación. No hubo diferencias significativas en la proporción de pacientes con cánceres hematológicos vs. proveedores de atención médica (10 vs. $7 \%$; $p=0.322$ ), pero hubo una diferencia significativa en la tasa de letalidad (62 vs. $0 \% ; p=0.002$ ). La mayoría de los pacientes tenían leucemia mieloide aguda (39\%) o linfoblástica aguda (20\%), el 59\% había recibido quimioterapia, con un intervalo medio desde el final del último ciclo de quimioterapia de 9 días (rango: 7-19 días)².

Mehta, et al. ${ }^{3}$ informaron de que entre 218 pacientes con cáncer y COVID-19 en un sistema de salud diferente hubo una tasa de mortalidad del $25 \%$ en pacientes con tumores sólidos y una tasa de mortalidad del $37 \%$ (68 muertes totales) en pacientes con neoplasias hematológicas.

En un artículo de Zhang, et al. ${ }^{4}$ se informó una asociación entre la terapia contra el cáncer en los últimos 14 días y los efectos graves de la infección por SARS-CoV-2 (hazard ratio [HR]: 4.079; intervalo de confianza del 95\% [IC 95\%]: 1.086-15.322; $p=0.037$ ) en 28 pacientes. Aunque estos datos son limitados, la interrupción del tratamiento contra el cáncer en pacientes con COVID-19 activa debe considerarse seriamente, ya que la continuación del tratamiento puede conducir a una mayor inmunosupresión y riesgo de complicaciones graves ${ }^{4}$.

\section{Métodos}

Analizamos datos de abril a junio de 2020. El estudio incluye a personas hospitalizadas en el Servicio de Hematología del Hospital General de México Dr. 
Eduardo Liceaga. Los cánceres hematológicos se clasificaron según la clasificación de la OMS de 2016 y se incluyeron patologías hematológicas benignas.

Se realizaron las pruebas cuantitativas de reacción en cadena de la polimerasa en tiempo real para el diagnóstico de infección por SARS-CoV-2, el diagnóstico de COVID-19 se basó inicialmente en parámetros clínicos, estudios de laboratorio y estudios de imagen (radiografía de tórax y tomografía computarizada [TC]).

La gravedad de la COVID-19 se calificó de la siguiente manera:

1- Leve: síntomas clínicos leves, sin neumonía en la TC pulmonar.

2- Moderada: fiebre, tos y TC pulmonar con neumonía

3- Grave: dificultad respiratoria (frecuencia respiratoria $>30$ minutos, saturación de oxígeno $\leq 93 \%$ en reposoy/o relación presión parcial de oxígeno arterial:oxígeno inspirado fraccional $\leq 300 \mathrm{mmHg}$.

4- Crítica: los criterios antes mencionados de insuficiencia respiratoria que reciben ventilación mecánica, shock y/o insuficiencia orgánica.

Todos los pacientes hospitalizados fueron transferidos a salas de aislamiento a los servicios de neumología, torre quirúrgica o terapias intensivas cuando fueron diagnosticados con COVID-19.

\section{Recopilación de datos}

Se realizó la recopilación prospectiva de datos epidemiológicos, clínicos y de laboratorio, los informes de radiología, los detalles de la terapia y los resultados en los pacientes se obtuvieron accediendo a registros médicos electrónicos y visitando a nuestros pacientes en las diversas áreas de hospitalización.

\section{Resultados}

Los resultados se muestran en las tablas 1-4.

\section{Características de los pacientes con patologías hematológicas y COVID-19}

Estudiamos 33 pacientes hospitalizados con patologías hematológicas. Predominó el sexo masculino $(n=20,60.6 \%)$ en comparación con el sexo femenino ( $n=13,39.4 \%$ ). En cuanto a la edad, la mínima fue 18y la máxima fue 72 (media: 36.94 años).

La mayoría de los pacientes hospitalizados tenían leucemia linfoide aguda $(n=13)$, leucemia mieloide
Tabla 1. Características generales de los pacientes con COVID19 y enfermedades hematológicas

\begin{tabular}{|l|c|}
\hline & COVID-19 n = 33 \\
\hline Sexo & \\
\hline Femenino & $13(39.4 \%)$ \\
\hline Masculino & $20(60.6 \%)$ \\
\hline Edad en años & \\
\hline Media: 36.94 (18-72) & \\
\hline Comorbilidades & \\
\hline No & $26(78.8 \%)$ \\
\hline Sí & $7(21.2 \%)$ \\
\hline Diabetes & $2(6.1 \%)$ \\
\hline HTA & $3(9.1 \%)$ \\
\hline ERC & $1(3 \%)$ \\
\hline Hipotiroidismo & $1(3 \%)$ \\
\hline Patologías hematológicas & \\
\hline Leucemia linfoblástica aguda & $13(39.4 \%)$ \\
\hline Leucemia mieloide aguda & $6(18.2 \%)$ \\
\hline Linfoma no Hodgkin & $6(18.2 \%)$ \\
\hline Síndromes mielodisplásicos & $1(3 \%)$ \\
Anemia aplásica & $3(9.1 \%)$ \\
Neoplasia de células dendríticas & $1(3 \%)$ \\
Leucemia mieloide crónica & $2(6.1 \%)$ \\
\hline Pancitopenia a estudio & $1(3 \%)$ \\
Hospitalización previa & $13(39.4 \%)$ \\
\hline Terapia previa & \\
\hline Quimioterapia & $17(51.5 \%)$ \\
\hline ATRA & $2(6 \%)$ \\
\hline Eltrombopag más ciclosporina & $2(6 \%)$ \\
\hline
\end{tabular}

COVID-19: enfermedad por coronavirus 2019; HTA: hipertensión arterial;

ERC: enfermedad renal crónica; ATRA: ácido transretinoico.

aguda $(n=6)$, linfoma no Hodgkin $(n=6)$, anemia aplásica $(n=3)$, síndrome mielodisplásico $(n=1)$, neoplasia de células dendríticas plasmocitoides blásticas $(n=1)$, leucemia mieloide crónica $(n=2)$ y pancitopenia a estudio $(n=1)$.

De aquellos con cáncer activo o patología hematológica benigna, en el momento del diagnóstico de COVID-19 4 (12.1\%) tenían enfermedad estable o que respondía, sin respuesta completa $24(72.7 \%)$, y con reciente diagnóstico/sin tratamiento 5 (15.2\%).

En general, los pacientes con cánceres hematológicos recibieron tratamiento anticancerígeno previo: quimioterapia 17 (51.5\%), los pacientes con leucemia promielocítica recibieron ATRA (ácido transretinoico) $(\mathrm{n}=2,6 \%$ ) y los pacientes con anemia aplásica recibieron eltrombopag más ciclosporina previo a diagnóstico por COVID-19 $(n=2,6 \%)$.

En nuestra población de estudio el $78.8 \%$ no presentaba comorbilidades.

Los pacientes con COVID-19 fueron tratados como se describe en la tabla 2 , el $100 \%$ de los pacientes recibieron antibióticos, el uso de hidroxicloroquina 
Tabla 2. Variables clínicas y de laboratorio, terapia y resultados de pacientes con patologías hematológicas y enfermedad por coronavirus 2019 (COVID-19)

\begin{tabular}{|l|c|}
\hline & COVID-19 $\mathbf{n = 3 3}$ \\
\hline Signos y síntomas al inicio & \\
\hline Fiebre & $28(85.71 \%)$ \\
\hline Tos & $20(61.8 \%)$ \\
\hline Disnea & $22(66.6 \%)$ \\
\hline Dolor muscular & $30(90.4 \%)$ \\
\hline Cefalea & $16(47.6 \%)$ \\
\hline Diarrea & $4(14.2 \%)$ \\
\hline Complicaciones & \\
\hline SDRA & $13(39.3 \%)$ \\
\hline Insuficiencia renal aguda & $3(9.5 \%)$ \\
\hline Septicemia & $9(23.8 \%)$ \\
\hline Tratamiento COVID-19 & \\
\hline Antibióticos & $33(100 \%)$ \\
\hline Hidroxicloroquina 27 & $(80.9 \%)$ \\
Oseltamivir 19 & $(57 \%)$ \\
\hline Enoxaparina & $9(27.27 \%)$ \\
\hline Oxígeno & $33(100 \%)$ \\
\hline Ventilación mecánica & $7(21.2 \%)$ \\
\hline Vivos & $15(45.46 \%)$ \\
\hline Fallecidos & $18(54.54 \%)$ \\
\hline SDRA: síndrome de dificultad respiratoria aguda. & \\
\hline
\end{tabular}

representó un $80.9 \%$, seguido de oseltamivir (57.1\%) y enoxaparina (27.27\%).

Entre la sintomatología reportada por los pacientes predominó la fiebre $(85.71 \%)$ y el dolor muscular (90.4\%).

Después del inicio de los síntomas de COVID-19, los pacientes con enfermedades hematológicas presentaban disminución de la cifra de hemoglobina, linfocitos y plaquetas y concentraciones, así como elevación de dímero $\mathrm{D}$ y ferritina.

Entre las principales complicaciones: con síndrome de dificultad respiratoria aguda 13 pacientes, 3 con disfunción renal aguda y 9 con sepsis.

El 100\% de los pacientes con patologías hematológicas y COVID-19 recibieron oxígeno suplementario, 7 pacientes en condición grave avanzaron a soporte de ventilación invasiva.

La mortalidad para los pacientes con patologías hematológicas fue del $54.54 \%$ (18 pacientes).

\section{Discusión}

Encontramos una tasa de casos de COVID-19 del $15.7 \%$ entre 210 pacientes hospitalizados con patologías hematológicas, mucho más alta que la reportada por $\mathrm{He}$, et al. ${ }^{2}$, quienes informaron que en un estudio de cohorte
Tabla 3. Valores de laboratorio al diagnóstico de COVID-19

\begin{tabular}{|c|c|}
\hline & Rango (Media) \\
\hline Hemoglobina (g/dl) & $3.6-12.7(7.9)$ \\
\hline Leucocitos (x 109/l) & $0.1-65(7.2)$ \\
\hline Neutrófilos (x 10/l) & $0-48.5(4.9)$ \\
\hline Linfocitos (x 109/l) & $0-12.7(1.1)$ \\
\hline Plaquetas (x 109/l) & 6-368 (93) \\
\hline Dímero D ( $\mu \mathrm{g} / \mathrm{l})$ & $162-24651(3065.7)$ \\
\hline TP segundos & $10.3-20.9(14)$ \\
\hline TTPa segundos & $20-47(30.5)$ \\
\hline TT segundos & $12.2-32.3(16.1)$ \\
\hline Fibrinógeno (mg/dl) & $0-1256(539.9)$ \\
\hline Ferritina (ng/ml) & $480-7270(2143.2)$ \\
\hline Troponina I (pg/ml) & $0.4-15.7(7.1)$ \\
\hline Proteína C reactiva $(\mathrm{mg} / \mathrm{dl})$ & $18.7-469(149)$ \\
\hline BNP (pg/ml) & $14.7-214.8(131.3)$ \\
\hline Procalcitonina (ng/ml) & $0.03-14.3(2.9)$ \\
\hline
\end{tabular}

Tabla 4. Estado de la enfermedad al diagnóstico de enfermedad por coronavirus 2019

\begin{tabular}{l|c}
\hline Respuesta completa/remisión & $4(12.1 \%)$ \\
\hline Sin respuesta completa & $24(72.7 \%)$ \\
\hline Reciente diagnóstico/sin tratamiento & $5(15.2 \%)$ \\
\hline Total & $33(100 \%)$ \\
\hline
\end{tabular}

de 128 pacientes con cánceres hematológicos aquellos con infección por COVID-19 representaban el 10\%.

Nuestros pacientes se caracterizaron en su mayoría por ser adultos jóvenes, sin comorbilidades en la mayoría, representando un $78.8 \%$.

Nuestro diagnóstico de COVID-19 se basó inicialmente en sintomatología referida: fiebre $(85.71 \%)$ y dolores musculares $(90.4 \%)$ fueron los principales síntomas referidos, asociados con estudios de laboratorios, entre lo cuales se destaca el valores de dímero $\mathrm{D}$ al momento del diagnóstico (con una media de 3,065 ug/l), con apoyo de estudios de imágenes radiográficas pulmonares. Todos los casos fueron confirmados por reacción en cadena de la polimerasa en tiempo real cuantitativa. 
La tasa de mortalidad de los pacientes hospitalizados con patologías hematológicas y COVID-19 fue del $54.54 \%$, en comparación con lo reportado por Mehta, et al. ${ }^{3}$, quienes informaron en 218 pacientes con cáncer y COVID-19 una tasa de mortalidad del $37 \%$ para cánceres hematológicos, y lo informado por $\mathrm{He}$, et al. ${ }^{2}$ sobre un estudio de cohorte de 128 pacientes con cánceres hematológicos con infección por COVID-19 con una tasa de letalidad del $62 \%$.

En nuestro estudio la principal patología hematológica fue leucemia linfoblástica aguda (39.4\%), seguida de linfoma no Hodgkin (18.2\%) y leucemia mieloide aguda (18.2\%), lo que difiere del estudio de $\mathrm{He}$, et al. ${ }^{2}$, en el que la mayoría de los pacientes tenían leucemia mieloide aguda (39\%) o linfoblástica aguda (20\%).

Encontramos pacientes hospitalizados con patologías hematológicas y con COVID-19 que tenían enfermedad más grave y con cáncer activo sin respuesta completa que recibieron quimioterapia citotóxica con un mayor riesgo de mortalidad por la COVID-19 en comparación con aquellos que no recibieron tratamiento activo, pacientes de novo sin quimioterapia, pero en estadios avanzados de la enfermedad con comorbilidades asociadas, lo que parece estar relacionado principalmente con coinfecciones bacterianas. Esto es consistente con una mayor probabilidad de disminución de las concentraciones de granulocitos debido a su enfermedad o la terapia de esta, ya que un 51.5\% habían recibido quimioterapia previamente. Esto es similar a lo publicado en el artículo de Zhang, et al. ${ }^{4}$, quienes informaron de una asociación entre la terapia contra el cáncer en los últimos 14 días y los efectos graves de la infección por COVID-19 (HR: 4.079; IC 95\%: 1.086-15.322; $p=0.037$ ) en 28 pacientes.

En general, al interpretar estos datos y ponerlos en contexto, sugerimos que continuar protegiendo a los pacientes con patologías hematológicas de la exposición al SARS-CoV-2 es importante, por medio del autoaislamiento, minimizando de forma segura el número de visitas al hospital, evitando contacto de pacientes COVID-19 negativos y COVID-19 positivos dentro del entorno hospitalario, y mitigando el riesgo de neutropenia para evitar el riesgo de COVID-19 simultáneo y septicemia bacteriana como se ha realizado en nuestro servicio de hematología.

\section{Conclusiones}

Los pacientes con patologías hematológicas muestran condiciones de deterioro y malos resultados de la infección con SARS-CoV-2. En nuestro estudio la mortalidad por COVID-19 parece deberse principalmente a enfermedad activa sin respuesta al tratamiento en estadios avanzados y enfermedad grave a su ingreso, no tanto a la edad, el sexo y las comorbilidades.

\section{Conflicto de intereses}

Los autores declaran que no existe conflicto de intereses.

\section{Financiamiento}

Los autores declaran que no existe financiamiento.

\section{Responsabilidades éticas}

Protección de personas y animales. Los autores declaran que para esta investigación no se han realizado experimentos en seres humanos ni en animales.

Confidencialidad de los datos. Los autores declaran que han seguido los protocolos de su centro de trabajo sobre la publicación de datos de pacientes.

Derecho a la privacidad y consentimiento informado. Los autores declaran que en este artículo no aparecen datos de pacientes.

\section{Bibliografía}

1. Zhu N, Zhang D, Wang W, Li X, Yang B, Song J, et al. A novel coronavirus from patients with pneumonia in China, 2019. N Engl J Med. 2020;382(8):727e733.

2. He W, Chen L, Chen L, Yuan G, Fang Y, Chen W, et al. COVID-19 in persons with haematological cancers. Leukemia. 2020;34(6):1637-45.

3. Mehta V, Goel S, Kabarriti R, Cole D, Goldfinger M, Acuna-Villaorduna A, et al. Case fatality rate of cancer patients with COVID-19 in a New York Hospital System. Cancer Discov. 2020;10(7):935-41.

4. Zhang L, Zhu F, Xie L, Wang C, Wang J, Chen R, et al. Clinical characteristics of COVID-19-infected cancer patients: a retrospective case study in three hospitals within Wuhan, China. Ann Oncol. 2020;31(7):894-901.

5. Dai M, Liu D, Liu M, Zhou F, Li G, Chen Z, et al. Patients with cancer appear more vulnerable to SARS-COV-2: a multi-center study during the COVID-19 outbreak. Cancer Discov. 2020;10(6):783-91.

6. Gale R. Perspective: SARS-CoV-2, COVID-19 and haematologists. Acta Haematol. 2020 May 6:1-4. doi: 10.1159/000508021. Online ahead of print.

7. Wang D, Hu B, Hu C, Zhu F, Liu X, Zhang J, et al. Clinical characteristics of 138 hospitalized patients with 2019 novel coronavirus-infected pneumonia in Wuhan, China. JAMA. 2020;323:1061-9.

8. Yu J, Ouyang W, Chua MLK, Xie C. SARS-CoV-2 transmission in patients with cancer at a tertiary care hospital in Wuhan, China. JAMA Oncol. 2020;6(7):1108-10.

9. Xia Y, Jin R, Zhao J, Li W, Shen H. Risk of COVID-19 for patients with cancer. Lancet Oncol. 2020;21:e180.

10. World Health Organization. Clinical management of severe acute respiratory infection when novel coronavirus (nCoV) infection is suspected. Interim Guidance [Internet]. Ginebra: World Health Organization; 2020. Disponible en: https://www.who.int/publications-detail/clinical-management-of-severeacute-respiratory-infection-when-novel-coronavirus-(ncov)-infectionis-suspected. Accessed April 4, 2020

11. Guan WJ, Ni ZY, Hu Y, et al. Clinical characteristics of coronavirus disease 2019 in China. N Engl J Med. 2020;382(18):1708-20.

12. Kuderer N, Choueiri T, Shah D, Shyr Y, Rubinstein S, Rivera D, et al. Clinical impact of COVID-19 on patients with cancer (CCC19): a cohort study. Lancet. 2020;395(10241):1907-18.

13. Lee L, Cazier J, Starkey T, Turnbull C, Kerr R, Middleton G. COVID-19 mortality in patients with cancer on chemotherapy or other anticancer treatments: a prospective cohort study. Lancet. 2020;395(10241):1919-26. 


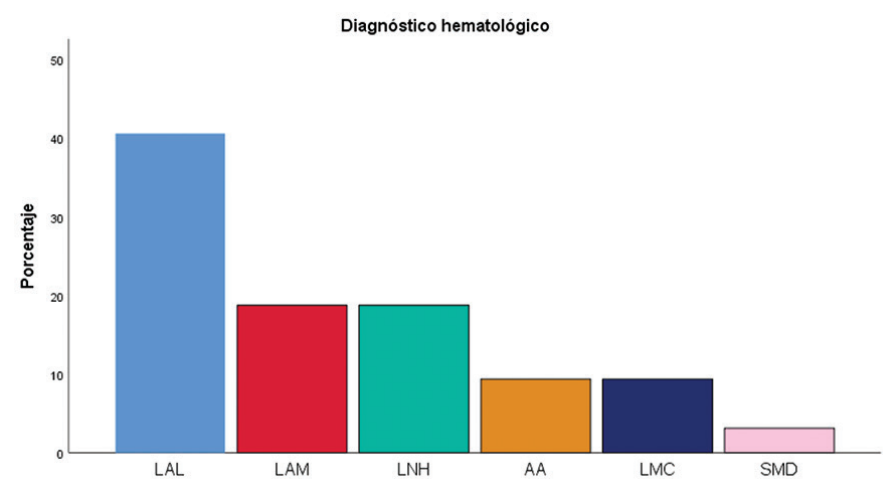

Sexo de paciente con patologías hematológicas -Covid-19

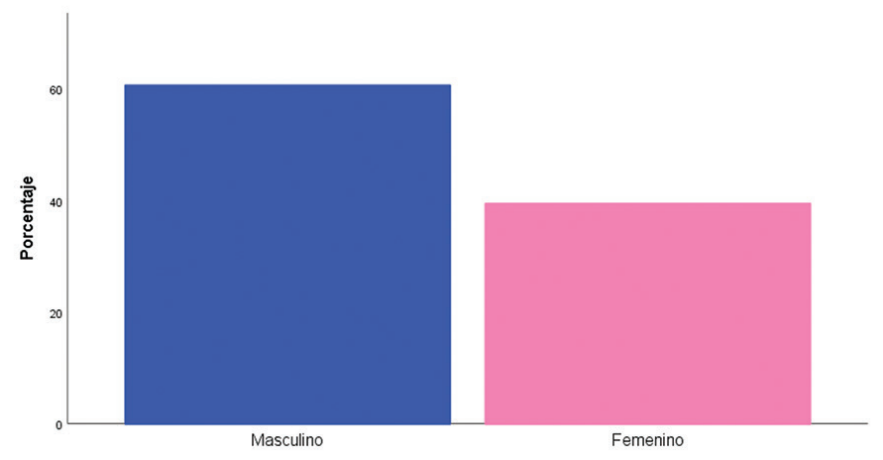

Comorbilidades de paciente con patologías hematológicas -Covid-19
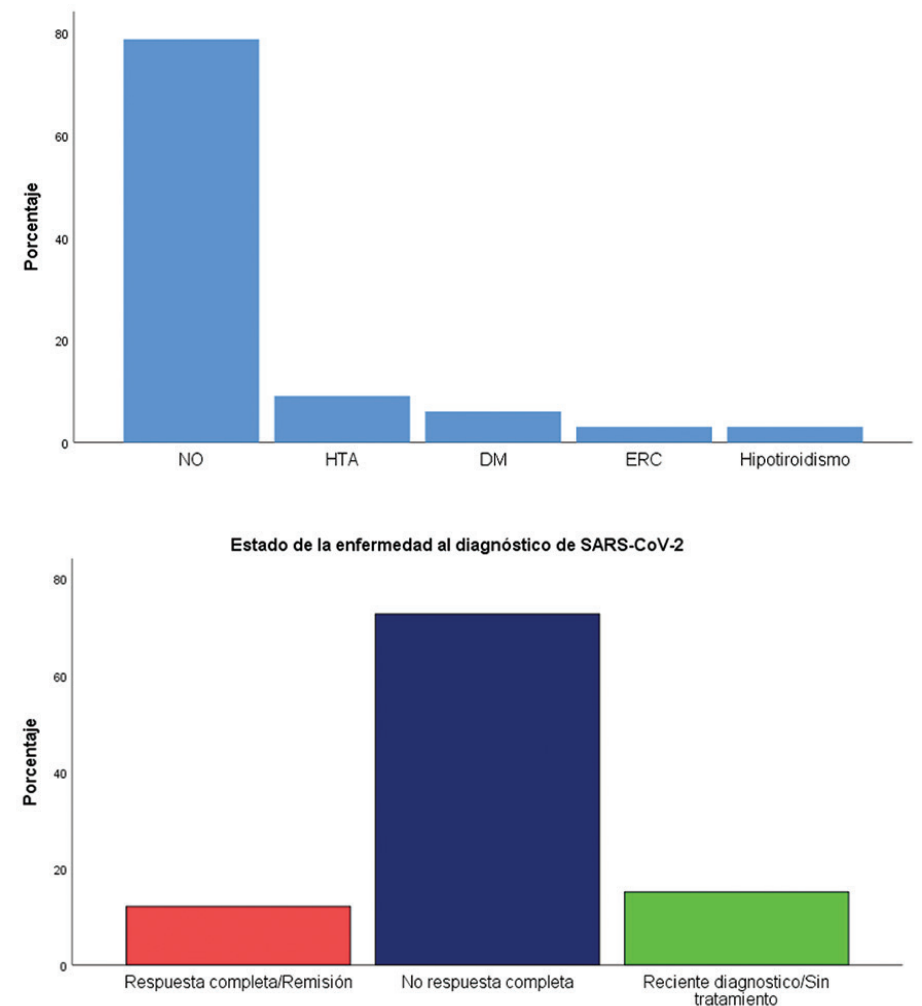

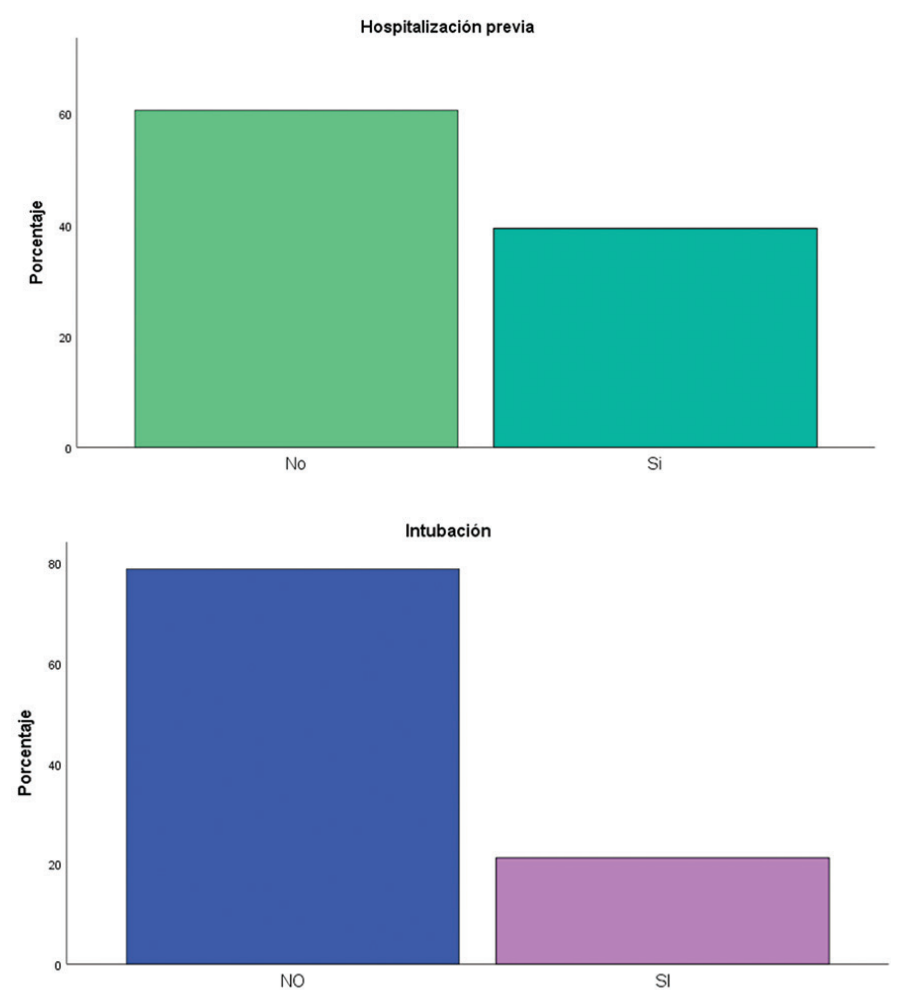

Egreso de paciente con patologías hematológicas -Covid-19
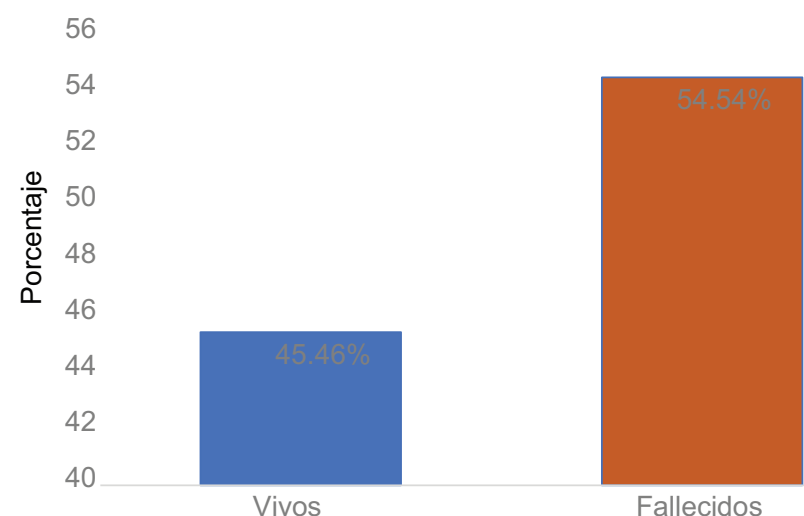

Fallecidos

Estudios de imágenes de pacientes con patologías hematológicas y Covid-19

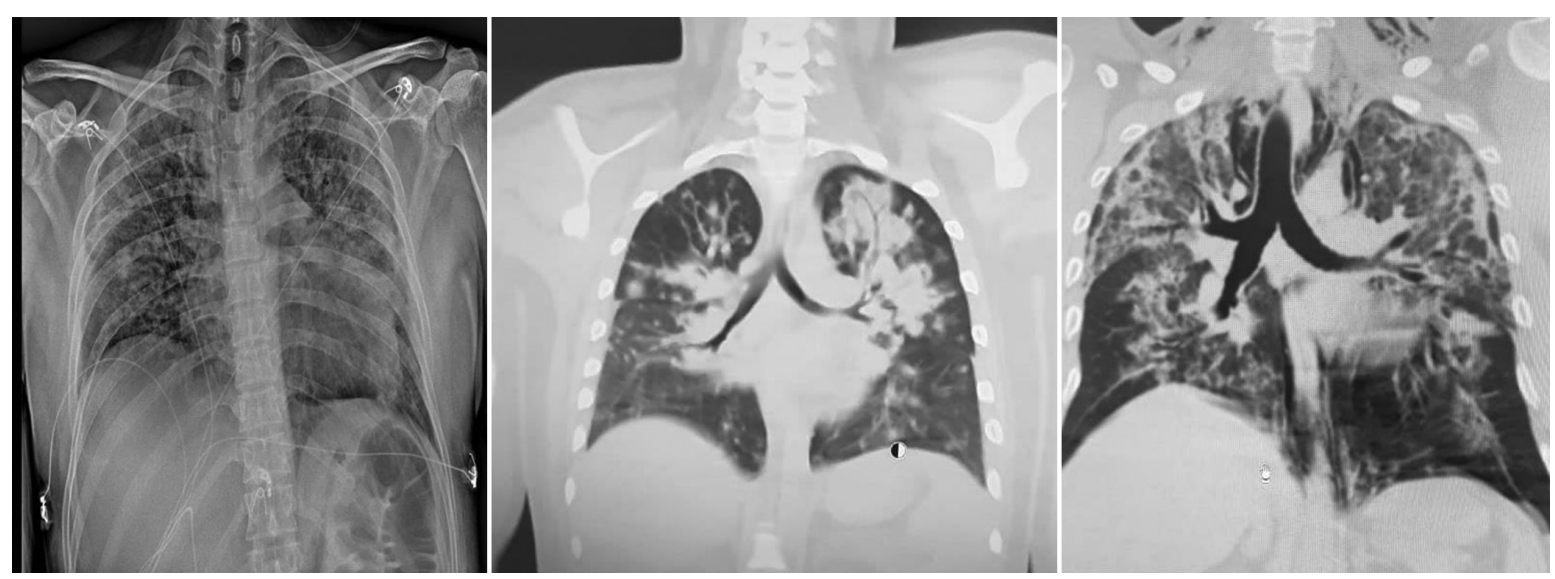

